\title{
Overexpression of human SODI improves survival of mice susceptible to endotoxic shock
}

This article was published in the following Dove Press journal:

Journal of Inflammation Research

23 July 2012

Number of times this article has been viewed

\author{
Jean Charchaflieh ${ }^{1,2}$ \\ Georges I Labaze' \\ Pulsar $\mathrm{Li}^{\prime}$ \\ Holly Van Remmen ${ }^{3}$ \\ Haekyung Lee' \\ Helen Stutz' \\ Arlan Richardson ${ }^{3}$ \\ Asher Emanuel' \\ Ming Zhang ${ }^{1,4}$ \\ 'Department of Anesthesiology, \\ State University of New York (SUNY) \\ Downstate Medical Center, \\ New York, NY, USA; ${ }^{2}$ Department of \\ Anesthesiology, Yale University School \\ of Medicine, New Haven, CT, USA; \\ ${ }^{3}$ Barshop Center for Longevity and \\ Aging Studies, University of Texas \\ Health Science Center at San Antonio, \\ San Antonio, TX, USA; ${ }^{4}$ Department \\ of Cell Biology, State University of \\ New York (SUNY) Downstate Medical \\ Center, New York, NY, USA
}

Correspondence: Ming Zhang Department of Anesthesiology, State University of New York (SUNY) Downstate Medical Center, 450 Clarkson Avenue, Brooklyn, NY II 203, USA

$\mathrm{Tel}+|7| 8270|4| 3$

Fax +I 7182703928

Email ming.zhang@downstate.edu
Background: Protective effects of the antioxidant enzyme Cu-Zn superoxide dismutase (SOD1) against endotoxic shock have not been demonstrated in animal models. We used a murine model to investigate whether overexpression of SOD1 protects against endotoxic shock, and whether the genetic background of SOD1 affects its effective protective effects and susceptibility to endotoxic shock.

Methods: Transgenic (tg) mice overexpressing human SOD1 and control mice were divided into four groups based on their genetic background: (1) tg mice with mixed genetic background (tg-JAX); (2) wild-type (WT) littermates of tg-JAX strain (WT-JAX); (3) tg mice with C57BL/6J background (tg-TX); (4) WT littermates of tg-TX strain (WT-TX). Activity of SOD1 in the intestine, heart, and liver of $\mathrm{tg}$ and control mice was confirmed using a polyacrylamide activity gel. Endotoxic shock was induced by intraperitoneal injection of lipopolysaccharide. Survival rates over 120 hours (mean, 95\% confidence interval) were analyzed using Kaplan-Meier survival curves.

Results: Human SOD1 enzymatic activities were significantly higher in the intestine, heart, and liver of both tg strains (tg-JAX and tg-TX) compared with their WT littermates (WT-JAX and WT-TX, respectively). Interestingly, the endogenous SOD1 activities in tg-JAX mice were decreased compared with their WT littermates (WT-JAX), but such aberrant changes were not observed in tg-TX mice. There was no difference in the survival time between tg-JAX and WTJAX groups after endotoxic shock $(P>0.05)$. However, the survival time in the tg-TX group was more than twofold longer than that in the WT-TX group $(P<0.05)$. In addition, WT-JAX mice survived significantly longer than WT-TX mice $(P<0.05)$.

Conclusion: Aberrant decrease of endogenous SOD1 activities may have overshadowed the effect of overexpression of SOD1 in tg mice (tg-JAX). Mice with C57BL/6J background (tg-TX) are more susceptible to lipopolysaccharide-induced endotoxic shock than those with mixed genetic background (tg-JAX). Overexpression of SOD1 is protective only in mice with C57BL/6J background (tg-TX).

Keywords: human SOD1 enzyme, endotoxic shock, transgenic mice, protective effect

\section{Introduction}

Sepsis is a critical condition initiated by infection that leads to a systemic inflammatory response. Septic shock, defined as sepsis-induced hypotension and hypoperfusion despite adequate fluid resuscitation, is associated with mortality rates approaching $50 \%$. Clinical studies have shown that the levels of oxidant activity and oxidation products are increased in septic patients, ${ }^{1}$ while the levels of antioxidants are decreased. ${ }^{2,3}$ Clinical trials are ongoing to determine whether antioxidant supplementation can reduce mortality in patients with septic shock. ${ }^{4}$ 
Oxidative stress can be initiated through the formation of reactive oxygen species such as the superoxide anion $\left(\mathrm{O}_{2}^{-}\right)$. The enzyme $\mathrm{Cu}-\mathrm{Zn}$ superoxide dismutase (SOD1) acts upon the free radical $\mathrm{O}_{2}^{-}$to form oxygen $\left(\mathrm{O}_{2}\right)$ and hydrogen peroxide $\left(\mathrm{H}_{2} \mathrm{O}_{2}\right)$. The enzyme catalase can further reduce $\mathrm{H}_{2} \mathrm{O}_{2}$ to $\mathrm{H}_{2} \mathrm{O}$ and $\mathrm{O}_{2}$. The superoxide anion, in vivo, is well known for its ability to react with nitric oxide (NO) to form peroxynitrite $\left(\mathrm{ONOO}^{-}\right)$, a potent cytotoxic and proinflamatory molecule. ${ }^{5}$ Additionally, peroxynitrite inactivates SOD1 by donating a nitrate group. ${ }^{5}$ Superoxide anions lead to the formation of chemotactic factors, cytokine release, lipid peroxidation, DNA damage, neurotransmitter and hormone inactivation, and cytotoxicity. ${ }^{5}$

In the setting of sepsis and septic shock, these effects can eventually lead to death. It would be expected that an increase in antioxidant level or the enhancement of antioxidant enzyme activity would decrease oxidative stress, leading to decreased inflammatory response and improved outcome. However, a recent publication by Meissner et al indicated that in a murine endotoxic shock model, a deficiency of SOD1 provided protection against the effects of lipopolysaccharide (LPS). ${ }^{6}$ In addition, de Vos et al found that overexpression of SOD1 failed to demonstrate protection in a murine endotoxic shock model. ${ }^{7}$

We conducted our study to examine SOD1 effects on LPS-induced endotoxic shock in mice and to determine whether genetic background of SOD1 affects susceptibility and survival in LPS-induced endotoxic shock.

\section{Materials and methods Transgenic mice strains overexpressing SODI}

Two independently generated strains of transgenic ( $\mathrm{tg}$ ) mice overexpressing the human SOD1 gene were used. One, generated by Charles Epstein, was obtained from the Jackson Laboratory (Bar Harbor, ME) and is designated tg-JAX in this study. ${ }^{8}$ The JAX strain has a first-generation mixed genetic background of C57BL/6 * SJL (approximately 50\% C57BL/6 and 50\% SJL; Jackson Laboratory stock \#002297). Note that the Jackson Laboratory has backcrossed the strain multiple times after we obtained the breeding mice, but our experiments used mice with the original mixed background. The second strain, obtained from Arlan Richardson at the University of Texas and designated tg-TX, ${ }^{9}$ was on C57BL/6J background.

Breeding and animal care were carried out according to Institutional Animal Care and Use Committee guidelines at State University of New York (SUNY) Downstate
Medical Center. The littermates of the $\operatorname{tg}$ mice were genotyped through the services of GeneTyper (New York, NY). Wild-type (WT) littermates specific for each strain were used as controls. All study animals were 12-weekold male mice. There were no statistical differences in body weight between (1) WT-JAX and tg-JAX mice (29 $\pm 3 \mathrm{~g}$ vs $26 \pm 2 \mathrm{~g}, P>0.05)$; (2) WT-TX and tg-TX mice ( $24 \pm 2 \mathrm{~g}$ vs $22 \pm 3 \mathrm{~g}, P>0.05)$; (3) tg-JAX and tg-TX mice $(26 \pm 2 \mathrm{~g}$ vs $22 \pm 3 \mathrm{~g}, P>0.05)$. However, the average body weight of WT-JAX mice was slightly higher than that of WT-TX mice $(29 \pm 3 \mathrm{~g}$ vs $24 \pm 2 \mathrm{~g} ; P<0.05)$.

\section{SODI activity analysis}

SOD1 activity was determined using a polyacrylamide activity gel, as previously described by Beauchamp and Fridovich. ${ }^{9,10}$ Briefly, the heart, liver, and intestine tissues were harvested and kept frozen at $-70^{\circ} \mathrm{C}$ until being analyzed. Frozen tissues were homogenized without thawing in ten volumes of ice-cold homogenization buffer $(10 \mathrm{mM}$ $\mathrm{KH}_{2} \mathrm{PO}_{4}, \mathrm{pH} 7.4,20 \mu \mathrm{M}$ ethylenediaminetetraacetic acid, $30 \mathrm{mM} \mathrm{KCl}$ ) using a polytron homogenizer. The homogenate was centrifuged at $13,600 \times \mathrm{g}$ in a microcentrifuge at $-4^{\circ} \mathrm{C}$ for 10 minutes. The supernatant was separated and used for measurement of enzyme activity. Extracts (heart lysates, $20 \mu \mathrm{g}$ of protein per sample; liver and intestinal lysates, $15 \mu \mathrm{g}$ of protein per sample) were separated on a $10 \%$ polyacrylamide gel that was subsequently incubated in a solution containing nitroblue tetrazolium, riboflavin, and tetramethylethylenediamine. The gel was photographed and the image analyzed using the ImageJ program (National Institutes of Health, Bethesda, MD) to quantify the intensity of the bands representing activity.

Band intensity $=($ Meanintensity of the band $\times$ Area of the band $)$ /Amount of protein loaded per sample

\section{Induction of endotoxic shock}

Fifty-nine mice were included in the study. Mice were injected intraperitoneally with LPS at a dose of $35 \mathrm{mg} / \mathrm{kg}$ body weight. The LPS, derived from Escherichia coli serotype 0127:B8 (Sigma-Aldrich, St Louis, MO), has been proven to be able to induce endotoxic shock in murine models. ${ }^{11}$ Pilot studies using WT-TX littermates found that a dose of $35 \mathrm{mg} / \mathrm{kg}$ yielded survival times between 24 and 48 hours, enabling accurate monitoring of animal outcome during experiments. After LPS injection, the animals were monitored for development of endotoxic shock syndrome. Irreversible shock was considered to have occurred when the mice were unable to drink, move about, or right themselves when rolled onto their sides. 
The monitoring was carried out every hour for the first 12 hours and every 8 hours thereafter for a 120-hour period following injection. Mice that survived for 120 hours were considered fully recovered and were killed by cervical dislocation. Mice were fed chow and water ad libitum.

\section{Statistical analyses}

Data were entered into an Excel database (Microsoft, Redmond, WA) and analyzed by SPSS statistical software (v 20; SPSS Inc, Chicago, IL). All data were expressed as means \pm standard deviation (SD). To analyze the quantitative data of the SOD1 activity, Levene's test was used to determine the homogeneity of variances. Statistical significance was assessed by one-way analysis of variance (ANOVA) followed by post hoc Bonferroni test (when the equality of variances assumption held) or Dunnett's T3 test (when the equality of variances was not met). A $P$-value $<0.05$ was considered significant. Survival times were analyzed by the Kaplan-Meier method, and the log-rank test was used to determine statistical significance. Censored data indicate animals that survived beyond 120 hours. Results are reported as means with $95 \%$ CI. An alpha value of less than 0.05 was considered statistically significant.

\section{Results \\ SODI activity}

To confirm SOD1 enzymatic activities were globally overexpressed in tg mice than their WT littermates, we analyzed different tissues in tg and WT mice. On the polyacrylamide activity gel, bands 1 and 2 represented endogenous SOD1 activities from the mice. Band 3 represented mixed activities from both endogenous and tg human SOD1. Bands 4 and 5 represented tg human SOD1 activities.

\section{SODI activity in heart}

In heart tissues, there were no significant differences among the band 1 intensities of WT-JAX, tg-JAX, WT-TX, and tg-TX mice $(84,243 \pm 14,651,66,872 \pm 14,638,77,599 \pm 5977$, and $69,040 \pm 7239$, respectively, ANOVA $P>0.05$ ) (Figure 1).

However, ANOVA found there was a statistical difference among band 2 intensities $(P<0.05)$. Post hoc analyses showed a significant decrease in band 2 intensities in tgJAX mice compared to WT-JAX (4443 \pm 2357 vs 96,714 \pm $15,179, P<0.05)$. In contrast, band 2 intensities in tg-TX mice were similar to those of WT-TX $(72,002 \pm 19,690 \mathrm{vs}$ $89,233 \pm 12,195, P>0.05)$.

There was a statistical difference among band 3 intensities of all four groups (ANOVA $P<0.05$ ). Post hoc analyses showed a significant increase in band 3 intensities in tg-JAX mice compared to WT-JAX (107,895 \pm 9819 vs $31,979 \pm 9051, P<0.05)$. Similarly, band 3 intensities in tg-TX mice were significantly increased compared with those of WT-TX $(134,418 \pm 20,110$ vs $31,872 \pm 7969, P<0.05)$.

There was a statistical difference among band 4 intensities of all four groups (ANOVA $P<0.05$ ). Post hoc analyses showed a significant increase in band 4 intensities in tg-JAX mice compared to WT-JAX $(125,825 \pm 3758$ vs $2009 \pm 232$, $P<0.05)$. Similarly, band 4 intensities in tg-TX mice were significantly increased compared with those of WT-TX $(100,555 \pm 10,047$ vs $3395 \pm 2960, P<0.05)$.

There was a statistical difference among band 5 intensities of all four groups (ANOVA $P<0.05$ ). Post hoc analyses showed a significant increase in band 5 intensities in tg-JAX mice compared to WT-JAX $(180,283 \pm 6547$ vs $6 \pm 10$, $P<0.05)$. Similarly, band 5 intensities in tg-TX mice were significantly increased compared with those of WT-TX $(39,163 \pm 13,070$ vs $27 \pm 38, P<0.05)$. Interestingly, band 5 intensities in tg-JAX mice were also significantly higher than those of $\operatorname{tg}$-TX $(P<0.05)$.

\section{SODI activity in liver}

Similar to heart, in liver tissues there were no significant differences among the band 1 intensities of WT-JAX, tg-JAX, WT-TX, and tg-TX mice $(28,330 \pm 7114,26,790 \pm 12,030$, $23,006 \pm 8502$, and 29,590 \pm 7573 , respectively, ANOVA $P>0.05$ ) (Figure 2).

ANOVA showed a statistical difference among band 2 intensities $(P<0.05)$. Post hoc analyses showed a significant decrease in band 2 intensities in tg-JAX mice compared to WT-JAX $(43,216 \pm 4520$ vs $140,791 \pm 34,711, P<0.05)$. In contrast, band 2 intensities in tg-TX mice were similar to those of WT-TX $(128,317 \pm 36,361$ vs $110,621 \pm 14,899, P>0.05)$.

There was a statistical difference among band 3 intensities of all four groups (ANOVA $P<0.05$ ). Post hoc analyses showed a significant increase in band 3 intensities in tg-JAX mice compared to WT-JAX $(134,572 \pm 204$ vs 91,224 \pm $12,987, P<0.05)$. Similarly, band 3 intensities in tg-TX mice were significantly increased compared with those of WT-TX $(145,622 \pm 12,332$ vs $82,176 \pm 10,175, P<0.05)$.

There was a statistical difference among band 4 intensities of all four groups (ANOVA $P<0.05$ ). Post hoc analyses showed a significant increase in band 4 intensities in tg-JAX mice compared to WT-JAX (135,523 \pm 7087 vs $9195 \pm 5247$, $P<0.05)$. Similarly, band 4 intensities in tg-TX mice were significantly increased compared with those of WT-TX $(133,496 \pm 7163$ vs $7321 \pm 5259, P<0.05)$. 

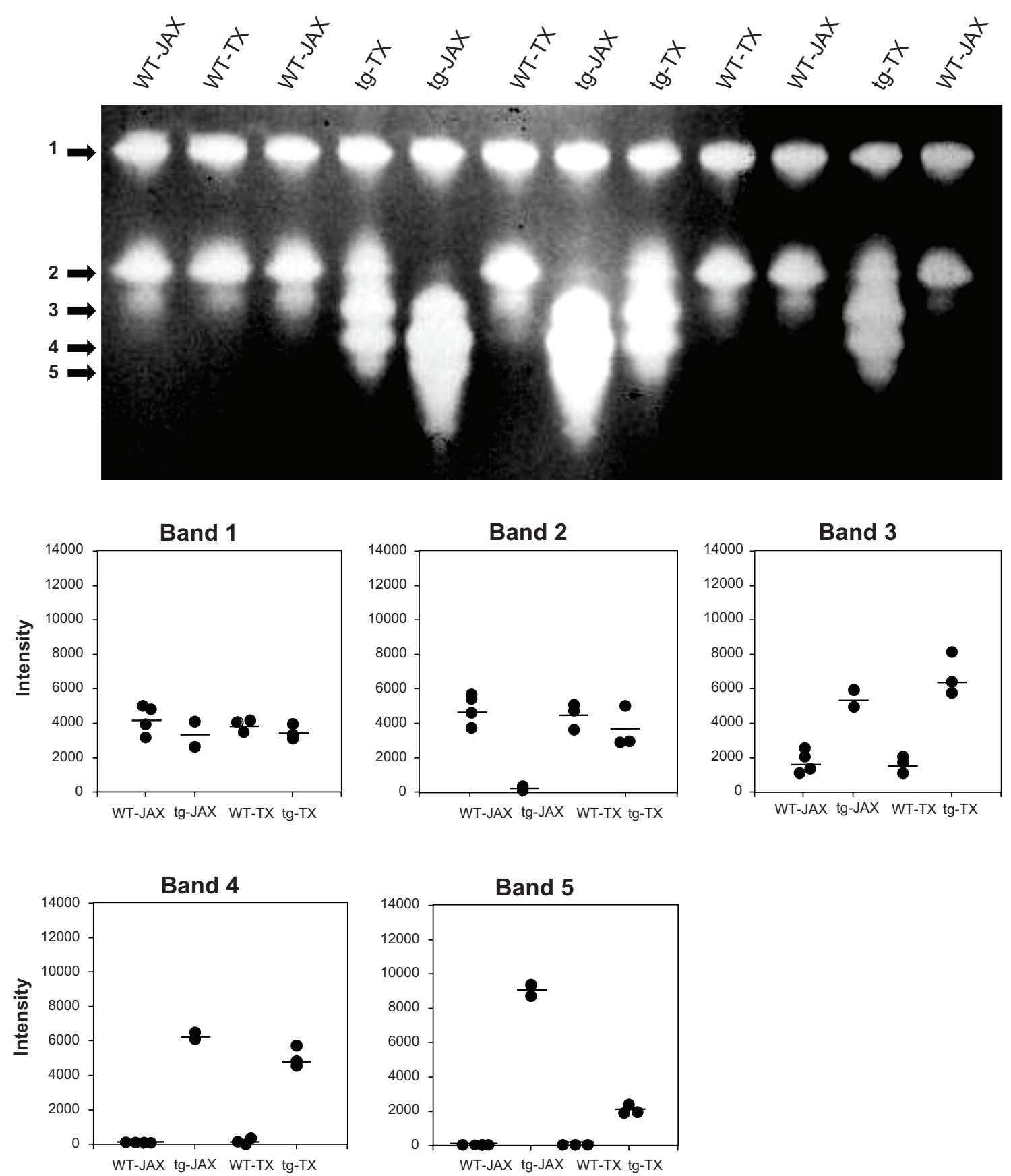

Figure I Cu-Zn superoxide dismutase (SODI) activity in heart tissues of two SODI transgenic (tg) strains and their littermates.

Notes: SODI activity was determined using a polyacrylamide activity gel as described in Materials and methods. Upper panel is the image of the activity gel. Each lane in the gels represents an individual mouse. The intensities of major bands of each mouse were quantified and summarized in the dot plots. The horizontal lines within the dot plots represent the average intensities of the respective strains of tg mice and their wild-type (WT) littermates. The asterisks indicate statistical significance $P<0.05$ between transgenic mice and their WT littermates.

There was a statistical difference among band 5 intensities of all four groups (ANOVA $P<0.05$ ). Post hoc analyses showed a significant increase in band 5 intensities in tg-JAX mice compared to WT-JAX $(174,010 \pm 3666$ vs $64 \pm 66$, $P<0.05)$. Similarly, band 5 intensities in tg-TX mice were significantly increased compared with those of WT-TX $(53,399 \pm 1336$ vs $10 \pm 14, P<0.05)$. In addition, band
5 intensities in tg-JAX mice were also significantly higher than those of $\operatorname{tg}-\mathrm{TX}(P<0.05)$.

\section{SOD I activity in intestinal tissues}

Similar to heart and liver, in intestinal tissues there were no significant differences among the band 1 intensities of WT-JAX, tg-JAX, WT-TX, and tg-TX mice (5641 \pm 1542 , 

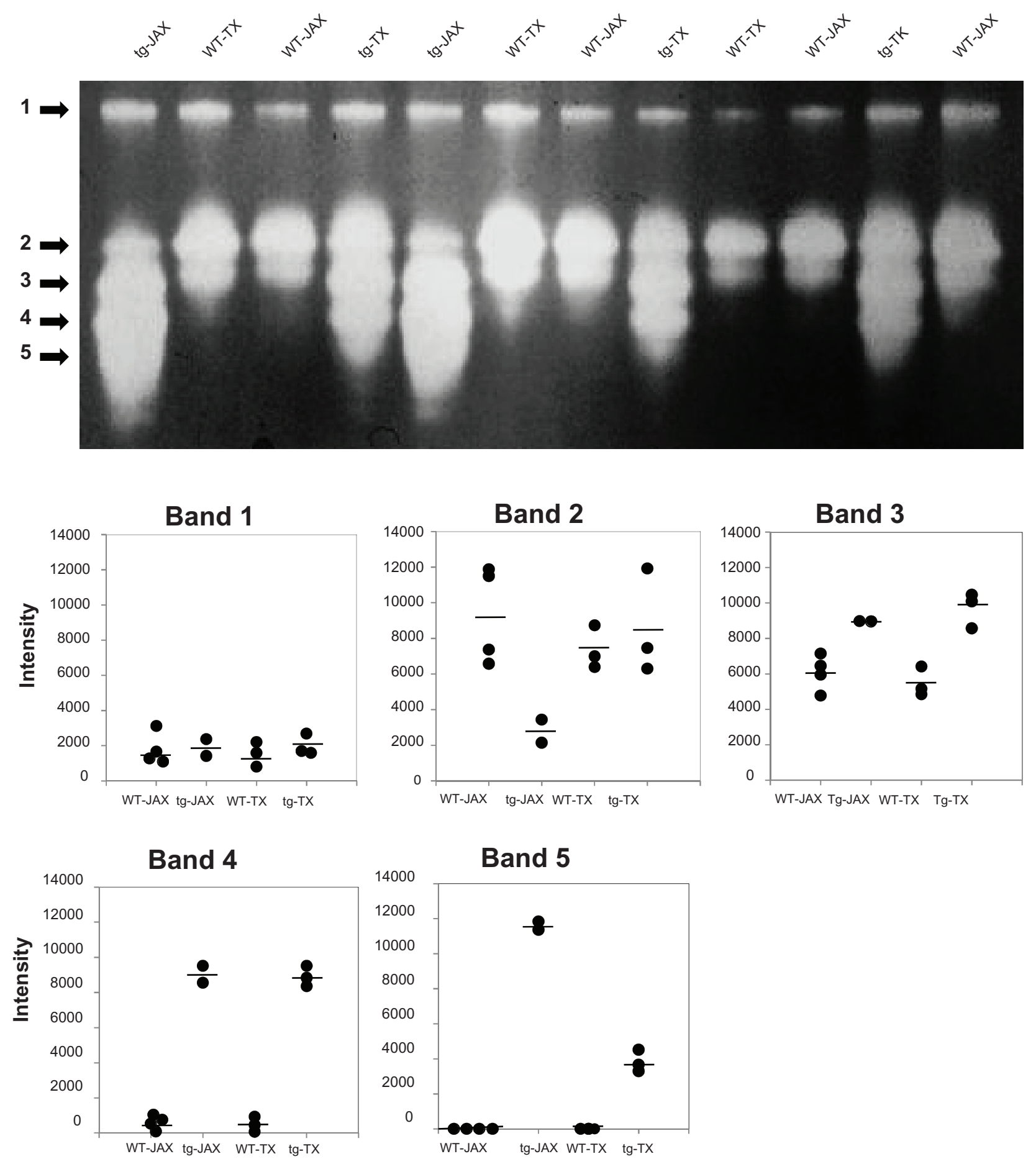

Figure $2 \mathrm{Cu}-\mathrm{Zn}$ superoxide dismutase (SODI) activity in liver tissues of two SODI transgenic (tg) strains and their littermates.

Notes: SODI activities in livers were determined as described in Figure I. The asterisks indicate statistical significance of $P<0.05$ between tg mice and their wild-type (WT) littermates.

$4685 \pm 1265,3567 \pm 1121$, and $4525 \pm 1885$, respectively, ANOVA $P>0.05$ ) (Figure 3).

ANOVA showed a statistical difference among band 2 intensities $(P<0.05)$. Post hoc analyses showed a significant decrease in band 2 intensities in tg-JAX mice compared to WT-JAX $(3045 \pm 1491$ vs 77,300 $\pm 11,830, P<0.05)$.
In contrast, band 2 intensities in tg-TX mice were similar to those of WT-TX $(63,489 \pm 6829$ vs $74,622 \pm 9900$, $P>0.05)$.

There was a statistical difference among band 3 intensities of all four groups (ANOVA $P<0.05$ ). Post hoc analyses showed a significant increase in band 3 intensities in tg-JAX 


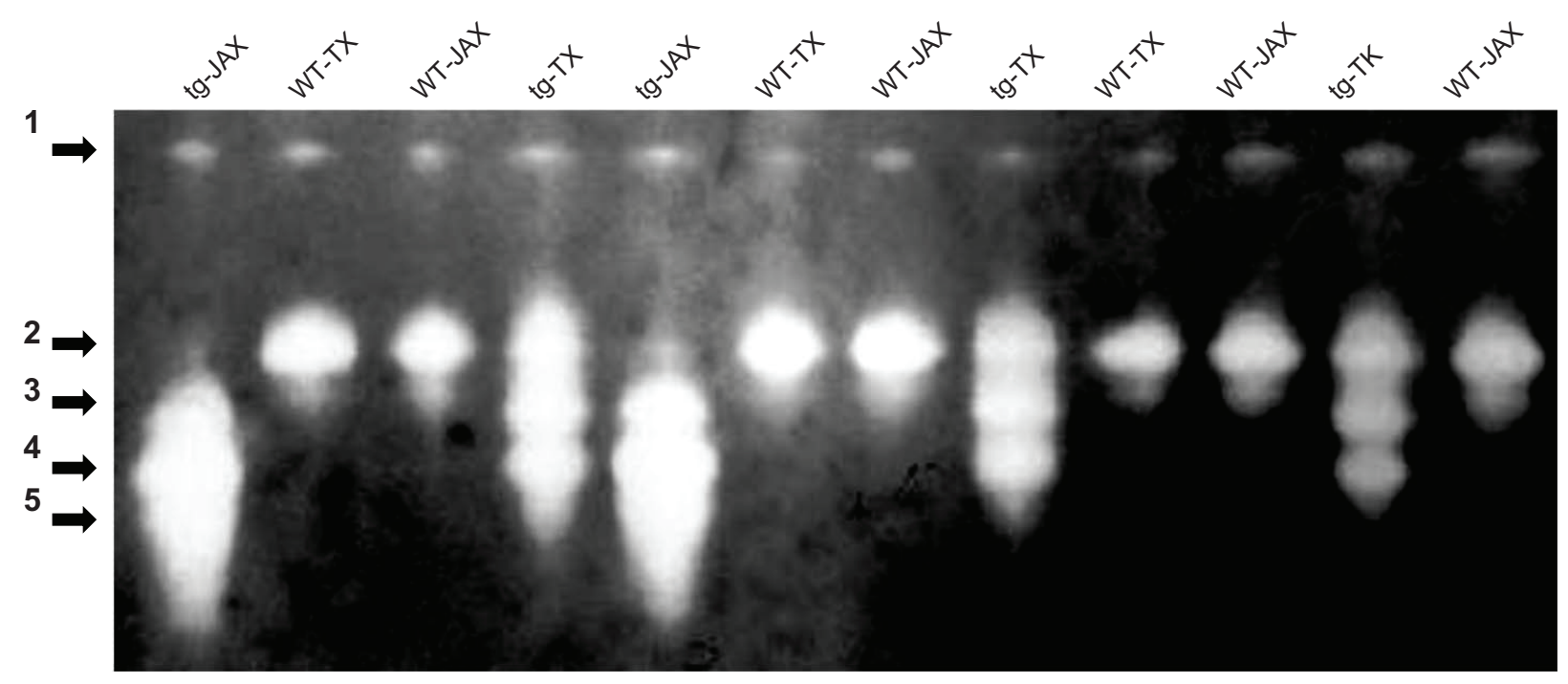

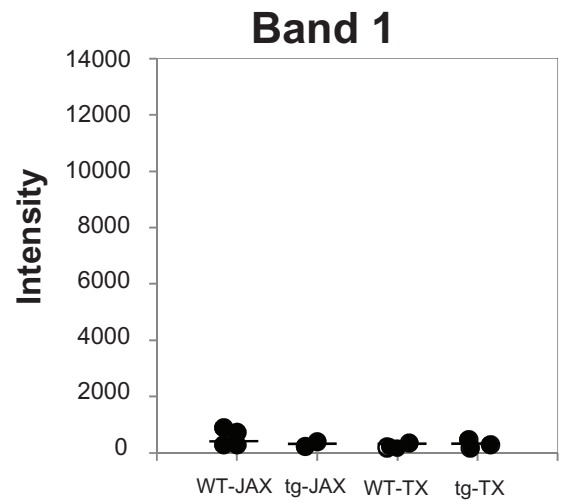

Band 4

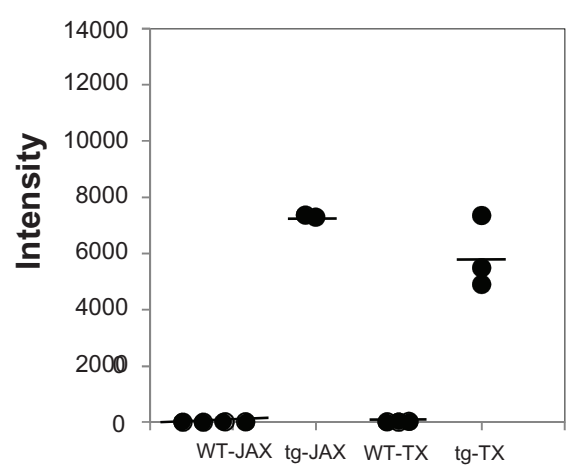

Band 2

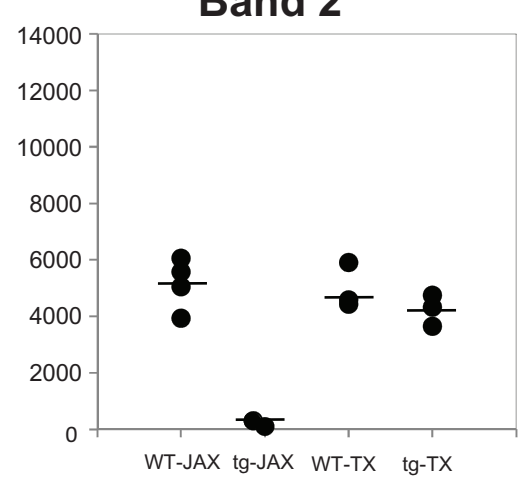

Band 5

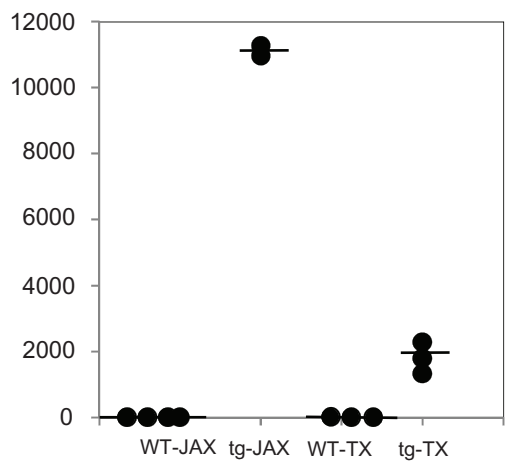

Figure $3 \mathrm{Cu}-Z n$ superoxide dismutase (SODI) activity in intestinal tissues of two SODI transgenic (tg) strains and their littermates.

Notes: SODI activities in intestines were determined as described in Figure I. The asterisks indicate statistical significance $P<0.05$ between tg mice and their wild-type (WT) littermates.

mice compared to WT-JAX $(94,366 \pm 2730$ vs $27,261 \pm$ 15,305, $P<0.05)$. Similarly, band 3 intensities in tg-TX mice were significantly increased compared with those of WT-TX $(96,380 \pm 5664$ vs 11,961 $\pm 5174, P<0.05)$.

There was a statistical difference among band 4 intensities of all four groups (ANOVA $P<0.05$ ). Post hoc analyses showed a significant increase in band 4 intensities in tg-JAX mice compared to WT-JAX (109,872 \pm 504 vs $86 \pm 53, P<0.05)$. Similarly, band 4 intensities in tg-TX mice were significantly increased compared with those of WT-TX $(88,674 \pm 15,733$ vs $196 \pm 189, P<0.05)$. There was a statistical difference among band 5 intensities of all 
four groups (ANOVA $P<0.05$ ). Post hoc analyses showed a significant increase in band 5 intensities in tg-JAX mice compared to WT-JAX $(166,671 \pm 2349$ vs $14 \pm 15, P<0.05)$. Similarly, band 5 intensities in tg-TX mice were significantly increased compared with those of WT-TX $(26,949 \pm 5822$ vs $75 \pm 106, P<0.05)$. Moreover, band 5 intensities in tgJAX mice were also significantly higher than those of $\operatorname{tg}$-TX $(P<0.05)$.

\section{Survival time after endotoxic shock}

tg-JAX and tg-TX mice (human SOD1 gene overexpressed on $\mathrm{C} 57 \mathrm{BL} / 6 * \mathrm{SJL}$ and $\mathrm{C} 57 \mathrm{BL} / 6$ genetic backgrounds, respectively), together with their WT littermates (WT-JAX and WT-TX, respectively), were subjected to endotoxic shock induced by LPS intraperitoneally at a dose of $35 \mathrm{mg} / \mathrm{kg}$ (Figure 4).

There was no statistical difference $(P>0.05)$ between the average survival times of the tg-JAX mice (42.3 hours, 95\% CI, range 27.2-57.4 hours, $\mathrm{n}=9$ ) and their WT littermates (WT-JAX; 36.8 hours, 95\% CI, range 27.2-45.9 hours, $n=24$ ) (Figure 4A). However, the tg-TX mice had an average survival time twofold greater than that of their WT littermates (WT-TX; 51.6 hours, 95\% CI, range 31-69 hours, $\mathrm{n}=17$, versus 24.7 hours, $95 \% \mathrm{CI}$, range $21.1-27.9$ hours, $\mathrm{n}=15$, respectively), shown to be statistically significant $(P<0.05)$ (Figure 4B).

The C57BL/6 * SJL mixed genetic-background WT littermates of the JAX tg strain had a longer average survival time than the $\mathrm{C} 57 \mathrm{BL} / 6$ genetic-background WT littermates of the TX $\operatorname{tg}$ strain $(P<0.05)$.

A

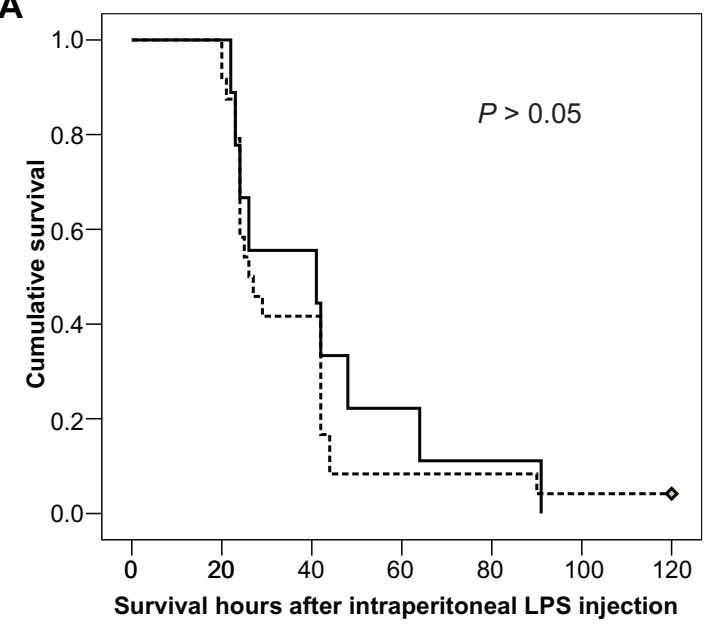

There was no statistically significant difference between the mean survival times of the tg mice from JAX and TX strains $(P>0.05)$.

\section{Discussion}

The tg-JAX mice overexpressing SOD1 showed no significant improvement in survival against endotoxin challenge over their WT littermates (WT-JAX) (Figure 4A), while the tg-TX mice survived significantly longer than their WT controls (WT-TX) (Figure 4B).

There was no statistically significant difference in survival times when the two tg strains were compared directly. However, comparison of the survival times of the WT littermates from two strains showed that those with the genetic background of JAX (WT-JAX) survived significantly longer than the WT-TX ones.

In tg mice from both JAX and TX strains, band intensities representing human SOD1 activity were significantly higher in heart (Figure 1, Bands 3, 4, and 5), liver (Figure 2, Bands 3, 4, and 5), and intestinal tissues (Figure 3, Bands 3, 4, and 5) than in the tissues of their WT littermates $(P<0.05)$. Surprisingly, some bands representing endogenous SOD1 activities in tg-JAX mice were aberrant. Although band 1 intensities were similar between both $\operatorname{tg}$ strains and their WT littermates $(P>0.05)$, band 2 intensities were significantly decreased in all tissues of tg-JAX mice compared with their WT littermates (Figures 1-3) $(P<0.05)$. Such aberrant changes were not observed in tissues of tg-TX mice. It is possible that aberrant decrease in endogenous SOD1 activities might have overshadowed the effect of overexpression of SOD1 in tg-JAX mice.

B

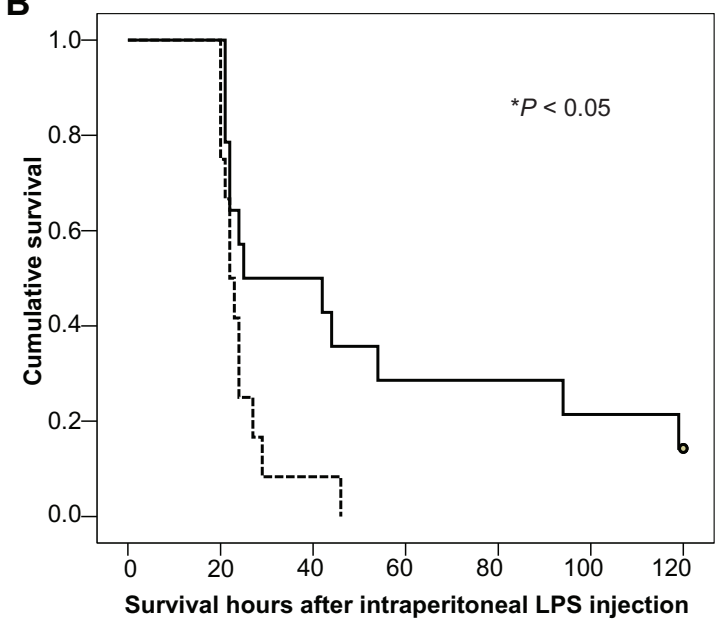

Figure 4 Survival analyses of two Cu-Zn superoxide dismutase (SODI) transgenic (tg) strains after endotoxic shock. SODI tg and wild-type (WT) mice were subjected to intraperitoneal injection of LPS. (A) Survival curves for tg-JAX mice (solid line) and their WT littermates (dotted line). The diamond represents censored data. (B) Survival curves for tg-TX mice (solid line) and their WT litermates (dotted line).

Notes: The circle and diamond represent censored data. The asterisks indicate statistically significant differences in survival curves $(P<0.05)$. 
Another explanation for the survival results is that the $\mathrm{C} 57 \mathrm{BL} / 6 \mathrm{~J}$ genetic background of the TX strain (tg-TX) gave the WT animals (WT-TX) an increased sensitivity to LPS, which was counteracted by overexpression of SOD1. WT animals with the 50\% C57BL/6J (mixed with 50\% SJL) genetic background (WT-JAX) were less sensitive to the effects of LPS than animals with the higher C57BL/6 genetic background (WT-TX). Overexpression of SOD1 in the mixed-genetic-background mice (tg-JAX) did not improve the survival times. Thus, the genetic background on which the gene for the SOD1 protein is placed is important when evaluating the effectiveness of scavenging superoxide anions on endotoxin-induced shock.

The results are relevant to the two reports cited earlier. Meissner et al showed that mice deficient in SOD1 were resistant to the effects of LPS-induced endotoxic shock, ${ }^{6}$ and de Vos et al showed that overexpression of SOD1 did not protect mice from LPS-induced endotoxic shock. ${ }^{7}$ The SOD1deficient mice of Meissner's study were from the Jackson Laboratory and had an incompletely specified C57BL/6 background $^{6}$ (the fact sheet from the Jackson Laboratory states that the strain was maintained on a mixed C57BL/6* 129S7 background at the time of Meissner's publication). The WT control mice in Meissner's study were age-matched female mice of unspecified genetic background, which were also used as controls for tg mice deficient in a different gene and maintained on a different genetic background. In the case of the de Vos study, mice overexpressing human SOD1 and their WT controls were clearly of different genetic backgrounds. Our carefully designed study showing that mice with different genetic backgrounds respond differently to LPS casts doubt on the conclusions of these studies.

Possible mechanisms for protection against endotoxic shock by overexpression of SOD1 include the ability to block the detrimental effects of free radicals on a large number of vital processes, including energy-generating pathways, survival/stress responses, apoptosis, the inflammatory response, and oxygen sensing. ${ }^{5}$

In summary, our study suggests that the benefits of antioxidant therapy during endotoxic shock depend on an individual's sensitivity to the oxidative stress-inducing agents. Further studies are needed to develop assays for determining

Journal of Inflammation Research

\section{Publish your work in this journal}

The Journal of Inflammation Research is an international, peer-reviewed open-access journal that welcomes laboratory and clinical findings on the molecular basis, cell biology and pharmacology of inflammation including original research, reviews, symposium reports, hypothesis formation and commentaries on: acute/chronic inflammation; mediators of inflamma- such sensitivities. Potential clinical applications include the provision of supplemental antioxidants to those septic-shock patients who show high sensitivity to LPS.

\section{Acknowledgments}

The authors would like to thank Dr James Cottrell for his continued support through Brooklyn Anesthesia Research and Dr Julie Rushbrook for critically reviewing the manuscript. The research was funded in part by NIH grant 1R21HL088527 (Zhang) and a SUNY-Downstate Dean's Award (Zhang).

\section{Disclosure}

The authors declare no conflicts of interest in this work.

\section{References}

1. Motoyama T, Okamoto K, Kukita I, Hamaguchi M, Kinoshita Y, Ogawa H. Possible role of increased oxidant stress in multiple organ failure after systemic inflammatory response syndrome. Crit Care Med. 2003;31:1048-1052.

2. Goode HF, Cowley HC, Walker BE, Howdle PD, Webster NR. Decreased antioxidant status and increased lipid peroxidation in patients with septic shock and secondary organ dysfunction. Crit Care Med. 1995;23:646-651.

3. Cowley HC, Bacon PJ, Goode HF, Webster NR, Jones JG, Menon DK. Plasma antioxidant potential in severe sepsis: a comparison of survivors and nonsurvivors. Crit Care Med. 1996;24:1179-1183.

4. Biesalski HK, McGregor GP. Antioxidant therapy in critical care - is the microcirculation the primary target? Crit Care Med. 2007;35: S577-S583.

5. Muscoli C, Cuzzocrea S, Riley DP, et al. On the selectivity of superoxide dismutase mimetics and its importance in pharmacological studies. $\mathrm{Br}$ J Pharmacol. 2003;140:445-460.

6. Meissner F, Molawi K, Zychlinsky A. Superoxide dismutase 1 regulates caspase-1 and endotoxic shock. Nat Immunol. 2008;9:866-872.

7. de Vos S, Epstein CJ, Carlson E, Cho SK, Koeffler HP. Transgenic mice overexpressing human copper/zinc-superoxide dismutase $(\mathrm{Cu} /$ $\mathrm{Zn}$ SOD) are not resistant to endotoxic shock. Biochem Biophys Res Commun. 1995;208:523-531.

8. Gurney ME, Pu H, Chiu AY, et al. Motor neuron degeneration in mice that express a human $\mathrm{Cu}, \mathrm{Zn}$ superoxide dismutase mutation. Science. 1994;264:1772-1775.

9. Chen X, Mele J, Giese H, et al. A strategy for the ubiquitous overexpression of human catalase and CuZn superoxide dismutase genes in transgenic mice. Mech Ageing Dev. 2003;124:219-227.

10. Beauchamp C, Fridovich I. Superoxide dismutase: improved assays and an assay applicable to acrylamide gels. Anal Biochem. 1971; 44:276-287.

11. Gadjeva M, Tomczak MF, Zhang M, et al. A role for NF-kappa B subunits p50 and p65 in the inhibition of lipopolysaccharide-induced shock. J Immunol. 2004;173:5786-5793.

\section{Dovepress}

tion; cellular processes; molecular mechanisms; pharmacology and novel anti-inflammatory drugs; clinical conditions involving inflammation. The manuscript management system is completely online and includes a very quick and fair peer-review system. Visit http://www.dovepress.com/ testimonials.php to read real quotes from published authors. 